
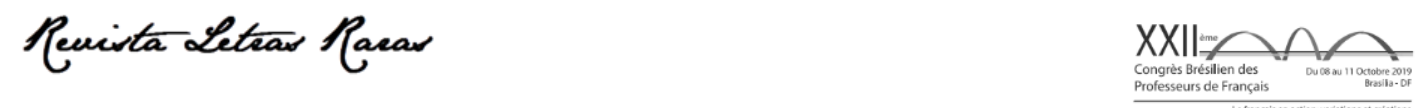

Actes du XXIlème Congrès brésilien des professeurs de français (dans Édition spéciale de la Revue Letras Raras 2020)

ISSN : 2317-2347 - v. 9, Dossier Spécial (2020)

Todo o conteúdo da RLR está licenciado sob Creative Commons Atribuição 4.0 Internacional

\title{
Le calque des pronoms personnels sujets et compléments: deux difficultés rencontrées lors de la traduction de 'L'Enfance d'un chef' (1939), de Sartre ${ }^{1}$
}

\section{Karol Garcia*}

Doctorat en Lettres - Littératures française et francophones, membre de l'équipe de recherche sur les difficultés de compréhension et/ou de traduction du français en portugais à l'UFRGS.

iD http://orcid.org/0000-0003-2312-2185

\section{Robert Ponge ${ }^{* *}$}

Professeur titulaire retraité de l'institut des lettres de l'UFRGS (Porto Alegre, RS, Brésil), professeur invité du centre d'études doctorales en lettres de la même université où il enseigne la littérature française et la traduction.

iD http://orcid.org/0000-0002-1078-8212

Reçu le10 oct. 2020. Approuvé le 30 oct. 2020.

Comment citer cet article :

GARCIA, Karol ; PONGE, Robert. Le calque des pronoms personnels sujets et compléments : deux difficultés rencontrées lors de la traduction de 'L'Enfance d'un chef' (1939), de Sartre. In: CONGRES BRESILIEN DES PROFESSEURS DE FRANÇAIS, 22., 2019, Brasília. Actes du XXIIème Congrès Brésilien des Professeurs de Français. Édition spéciale de la Revue Letras Raras: Campina Grande. EDUFCG. nov. 2020, p. 385-397.

\section{RÉSUMÉ}

Lors de la traduction en portugais brésilien de morceaux de "L'Enfance d'un chef », (1939), nouvelle de Sartre, nous avons connu diverses difficultés. Ce travail en examine deux, de nature lexico-syntaxique : la traduction du pronom personnel sujet «il(s)» et celle du pronom personnel complément d'objet indirect «lui». Nous les étudions dans deux extraits où leurs occurrences sont nombreuses. Après une rapide mise en contexte des deux passages, nous analysons le premier jet de notre traduction en portugais, expliquons comment nous avons perçu l'existence de problèmes, quel diagnostic nous avons fait et quelles solutions de traduction nous avons trouvées. Puis, nous cherchons à caractériser les écueils rencontrés, à comprendre leurs causes, à leur donner une dénomination commune

${ }^{1}$ Cet article reprend partiellement un travail précédent, publié en portugais par les auteurs. Le texte du présent article, en français, a été entièrement révisé, corrigé, revu et sa seconde moitié est substantiellement augmentée et reformulée. Le travail précédent se trouve dans : Anais do $7^{\circ}$ SENALLP e $1^{\circ}$ CIELLE. Rio Grande, RS: Editora da FURG, 2019. p. 261-274. Disponível em: https://senallp.furg.br/images/ANAIS-2019-site.pdf.

$\triangle$ garciakarol12@gmail.com

$\triangle$ r.ponge@ufrgs.br 

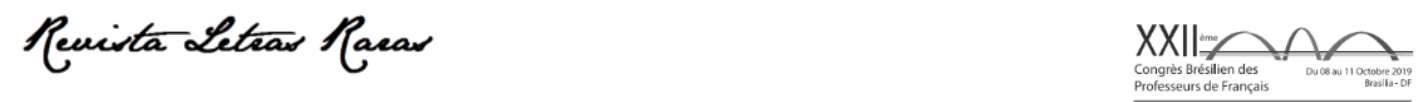

Actes du XXIlème Congrès brésilien des professeurs de français (dans Édition spéciale de la Revue Letras Raras 2020)

ISSN : 2317-2347 - v. 9, Dossier Spécial (2020)

Todo o conteúdo da RLR está licenciado sob Creative Commons Atribuição 4.0 Internacional

pour pouvoir les localiser dans une classification des types de difficultés de traduction. Nous terminons par une synthèse de notre parcours de recherche et des leçons que nous en avons tirées.

MOTS-CLÉS : Traduction ; Difficultés de traduction ; Calque; Jean-Paul Sartre.

\section{Introduction}

Ce texte a été élaboré dans le cadre de la thèse de doctorat en littérature de Karol Garcia et d'un projet de recherche sur les difficultés de compréhension et/ou de traduction du français en portugais, tous deux dirigés par Robert Ponge. La thèse contient, entre autres, une traduction partielle de "L'Enfance d'un chef » (1939), de Jean-Paul Sartre, et une étude de quelques difficultés rencontrées lors de la traduction en portugais du Brésil.

La méthodologie employée est bibliographique. Pour le concept de traduction, nous partons de celui de Dubois : «c'est énoncer dans une autre langue (ou langue cible) ce qui a été énoncé dans une langue source, en conservant», c'est-à-dire en cherchant à conserver «les équivalences sémantiques et stylistiques» (DUBOIS, 2002, p.487). Les autres bases théoriques et références bibliographiques utilisées sont mentionnées au cours de ce travail.

Nous étudions ici deux difficultés apparues dans les premières versions de notre traduction de la nouvelle de Sartre. Après une brève introduction, nous présentons notre projet de recherche et ses deux objectifs, nous exposons les deux problèmes perçus dans notre traduction, nous proposons des solutions et nous définissons à quel type de difficulté appartiennent les deux éléments examinés. Nous finissons par quelques commentaires conclusifs.

\section{Les deux objectifs du projet de recherche}

Le premier, théorique, est de réfléchir sur les types de difficultés de compréhension et/ou de traduction du français et d'en élaborer une classification. Pour ce faire, nous lisons d'abord des textes d'introduction (Mounin, 1971 ; Vinay \& Darbelnet, 1972 ; Rónai, 1976, 1977; Portinho, 1984; Durieux, 1999), puis des travaux ayant un rapport avec le sujet, surtout avec chaque type spécifique de difficulté: les faux amis, l'homonymie, la polysémie, le calque, les sens figurés, etc. L'objectif pratique veut organiser un glossaire alphabétique dont les articles rendent compte des principales difficultés concrètes de compréhension et/ou de traduction que la langue française 

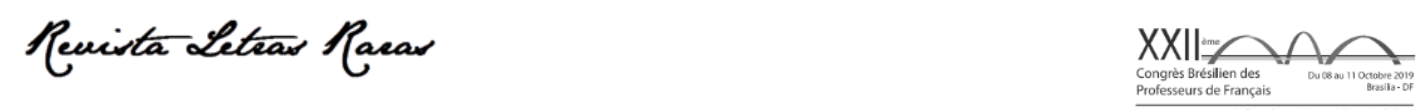

Actes du XXIlème Congrès brésilien des professeurs de français (dans Édition spéciale de la Revue Letras Raras 2020)

ISSN : 2317-2347 - v. 9, Dossier Spécial (2020)

Todo o conteúdo da RLR está licenciado sob Creative Commons Atribuição 4.0 Internacional

pose aux lusophones du Brésil (pour de plus amples détails sur le projet de recherche, consulter SILVA \& PONGE, 2012, p. 113-122).

Dans ce but, nous commençons par collationner les articles de trois petits dictionnaires brésiliens de difficultés de compréhension et/ou de traduction du français (RÓNAI, 1975 ; BATH \& BIATO, 1998 ; XATARA \& OLIVEIRA, 2008). Nous produisons aussi des articles relatifs à des difficultés rencontrées dans la pratique de nos étudiants, de nos collègues et dans la nôtre. C'est le cas du problème analysé dans les deux prochaines parties de ce travail. II est issu des extraits de «L'Enfance d'un chef » que nous avons traduits.

Ce récit conte l'histoire de Lucien Fleurier de sa petite enfance jusqu'au début de sa vie adulte, espace temporel qui commence peu avant la Première Guerre et finit au début de la Deuxième Guerre.

Nous nous limitons ici à deux extraits des morceaux traduits.

\section{Le premier extrait}

C'est le premier paragraphe du récit. Lucien, petit enfant, réfléchit sur l'image que les adultes ont de lui, en réaction au commentaire d'un ami de la famille, M. Bouffardier: «C'est une vraie petite fille, dit-il en souriant. Comment t'appelles-tu ? Jacqueline ? Lucienne ? Margot ?». Même s'il proteste : « Je m'appelle Lucien », la phrase de M. Bouffardier suscite en Lucien des soupçons et des craintes : «ll n'était plus sûr de ne pas être une petite fille: [...]; il avait peur que les gens ne décident tout d'un coup qu'il n'était plus un petit garçon [...]» (SARTRE, 1939, p. 151).

Commence alors l'extrait choisi. Son texte se trouve à gauche dans le tableau cidessous, et à droite, notre traduction dans un stade très initial.

Tableau 1

\begin{tabular}{|l|l|}
\hline \multicolumn{1}{|c|}{ Texte source: premier extrait } & \multicolumn{1}{|c|}{ Le premier jet de notre traduction } \\
\hline II [Lucien] n'était plus sûr de ne pas être une petite & Ele [Lucien] não estava mais seguro de não ser \\
fille [...]; il avait peur que les gens ne décident tout & uma menina [...]; ele temia que as pessoas \\
d'un coup qu'il n'était plus un petit garçon [...]. Tout & decidissem de repente que ele não era mais um \\
le monde lui dirait: ma jolie petite chérie; peut-être & menino [...]. Todo mundo Ihe diria, minha linda \\
que ça y est déjà, que je suis une petite fille; il se & queridinha; talvez já é assim, talvez já sou uma \\
sentait si doux en dedans, que c'en était un petit & menina; ele se sentia tão meigo por dentro que \\
peu écœurant, [...], et il offrit des fleurs à tout le & chegava a ser um pouco enjoativo, [...], e ele \\
monde avec des gestes arrondis, [...]. Il pensa: ça & ofereceu flores a todo mundo com gestos \\
\hline
\end{tabular}



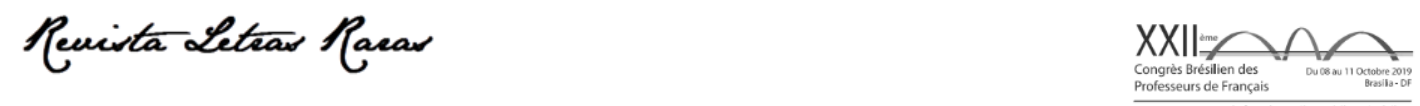

Actes du XXIlème Congrès brésilien des professeurs de français (dans Édition spéciale de la Revue Letras Raras 2020)

ISSN : 2317-2347 - v. 9, Dossier Spécial (2020)

Todo o conteúdo da RLR está licenciado sob Creative Commons Atribuição 4.0 Internacional

n'est pas pour de vrai. II aimait bien quand ça n'était pas pour de vrai mais il s'était amusé davantage le jour du Mardi gras: on l'avait costumé en Pierrot, il avait couru et sauté en criant, avec Riri, et ils s'étaient cachés sous les tables. arredondados, [...]. Ele pensou: isso é de faz de conta. Ele gostava quando era de faz de conta, mas ele tinha se divertido mais na terça-feira de carnaval quando o fantasiaram de Pierrot, ele tinha corrido e pulado e gritado, com Riri, e eles tinham se escondido sob as mesas.

Lorsque nous avons relu nos premiers brouillons, quelque chose a sonné bizarre dans notre traduction. Pendant le processus de révision, dans un dialogue fructueux avec les collègues de notre équipe de recherche, nous avons identifié que notre inconfort procédait, dans la version en portugais, de la grande quantité de pronoms personnels sujets de la troisième personne. Nous expliquons.

En français, le pronom personnel sujet accompagne toujours (ou presque toujours) le verbe. Pourquoi ? Parce que plusieurs désinences verbales françaises sont non distinctives phonétiquement (et même graphiquement), ce qui rend obligatoire l'usage de ce pronom, l'ellipse étant rare ou restreinte à quelques situations où le contexte et la syntaxe le permettent. II y a donc en français une présence permanente, systématique du pronom personnel sujet.

Cette contrainte n'existe pas en portugais où les pronoms personnels sujets sont souvent superflus, car les désinences verbales essentielles sont en général phonétiquement et graphiquement distinctives. Leur ellipse étant courante, c'est leur maintien qui sonne mal.

Revenons à notre inconfort. Pendant nos révisions, nous avons constaté qu'il procédait justement, dans la version en portugais, de la grande fréquence de pronoms personnels sujets masculins de la troisième personne, fréquence tellement répétitive qu'elle gênait. Cette récurrence provenait de la traduction machinale de tous les «il(s)» du texte français en « ele(s) » en portugais. C'est visible dans le deuxième tableau, ci-dessous, où sont soulignées les occurrences de «il(s)» dans la colonne de gauche, et les occurrences de « ele(s)» dans celle de droite:

Tableau 2

\begin{tabular}{|c|c|}
\hline Texte source: premier extrait & Le premier jet de notre traduction \\
\hline 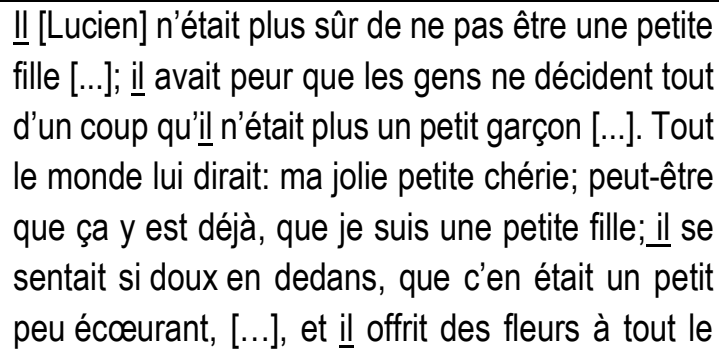 & $\begin{array}{l}\text { Ele [Lucien] não estava mais seguro de não ser } \\
\text { uma menina [...]; ele temia que as pessoas } \\
\text { decidissem de repente que ele não era mais um } \\
\text { menino [...]. Todo mundo lhe diria, minha linda } \\
\text { queridinha; talvez já é assim, talvez já sou uma } \\
\text { menina; ele se sentia tão meigo por dentro que } \\
\text { chegava a ser um pouco enjoativo, }[\ldots] \text {, e ele }\end{array}$ \\
\hline
\end{tabular}



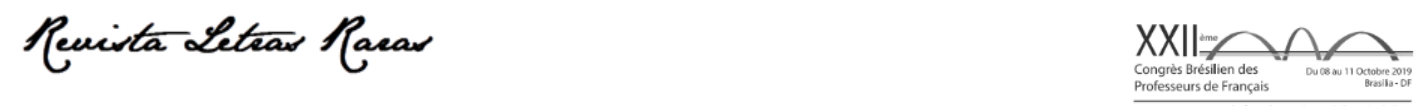

Actes du XXIlème Congrès brésilien des professeurs de français (dans Édition spéciale de la Revue Letras Raras 2020)

ISSN : 2317-2347 - v. 9, Dossier Spécial (2020)

Todo o conteúdo da RLR está licenciado sob Creative Commons Atribuição 4.0 Internacional

monde avec des gestes arrondis, [...]. ॥. pensa: ça n'est pas pour de vrai. II aimait bien quand ça n'était pas pour de vrai mais il s'était amusé davantage le jour du Mardi gras: on l'avait costumé en Pierrot, il avait couru et sauté en criant, avec Riri, et ils s'étaient cachés sous les tables. ofereceu flores a todo mundo com gestos arredondados, [...]. Ele pensou: isso é de faz de conta. Ele gostava quando era de faz de conta, mas ele tinha se divertido mais na terça-feira de carnaval quando o fantasiaram de Pierrot, ele tinha corrido e pulado e gritado, com Riri, e eles tinham se escondido sob as mesas.

Pour éliminer l'impression d'étrangeté résultant de la répétition de « ele(s)», nous avons supprimé plusieurs occurrences de ce pronom. Dans la colonne de droite du troisième tableau, les suppressions sont signalées par le symbole du vide (zéro barré) et les cas de maintien sont soulignés.

Tableau 3

\begin{tabular}{|c|c|}
\hline Texte source: premier extrait & Nouvelle version de notre traduction \\
\hline 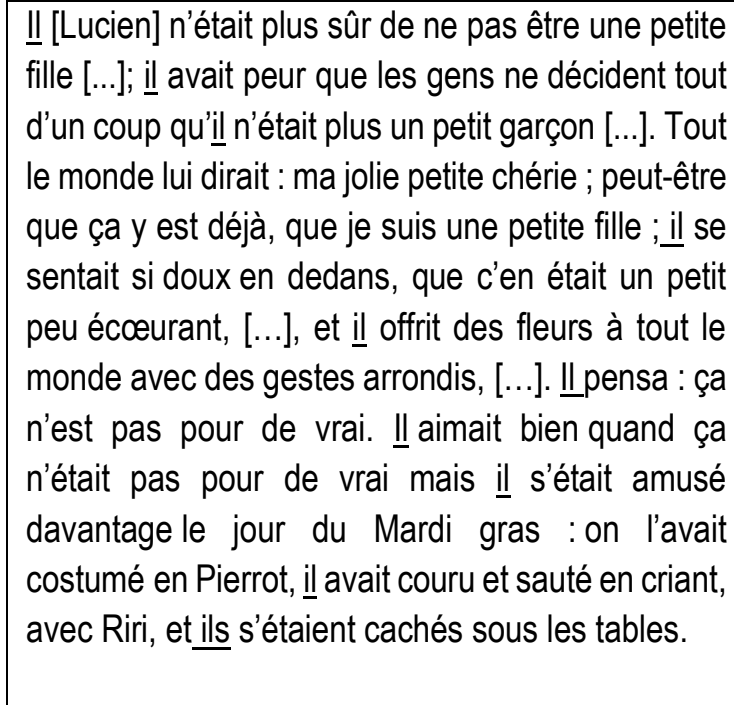 & $\begin{array}{l}\text { Ele [Lucien] não estava mais seguro de não ser } \\
\text { uma menina [...]; [Ø] temia que as pessoas } \\
\text { decidissem de repente que ele não era mais um } \\
\text { menino [...]. Todo mundo Ihe diria, minha linda } \\
\text { queridinha; talvez já é assim, talvez já sou uma } \\
\text { menina; [Ø] sentia-se tão meigo por dentro que } \\
\text { chegava a ser um pouco enjoativo, [...], e ele } \\
\text { ofereceu flores a todo mundo com gestos } \\
\text { arredondados, [...]. [Ø] Pensou: isso é de faz de } \\
\text { conta. Ele gostava quando era de faz de conta, } \\
\text { mas [Ø] tinha se divertido mais na terça-feira } \\
\text { de carnaval quando o fantasiaram de Pierrot, [Ø] } \\
\text { tinha corrido e pulado e gritado, com Riri, e [Ø] } \\
\text { tinham se escondido sob as mesas. }\end{array}$ \\
\hline
\end{tabular}

Nous passons maintenant au...

\section{Deuxième extrait}

Situé au début du troisième paragraphe de la nouvelle, il se passe quelques jours après celui du premier paragraphe. C'est l'heure du coucher. Lucien écoute l'histoire que raconte sa mère.

Dans le quatrième tableau, le texte source est à gauche et notre premier jet à droite.

Tableau 4

\begin{tabular}{|c|c|}
\hline Texte source: deuxième extrait & Le premier jet de notre traduction \\
\hline
\end{tabular}



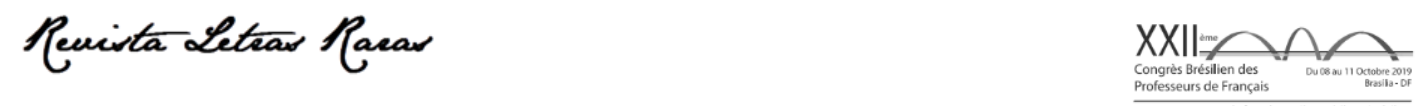

Actes du XXIlème Congrès brésilien des professeurs de français (dans Édition spéciale de la Revue Letras Raras 2020)

ISSN : 2317-2347 - v. 9, Dossier Spécial (2020)

Todo o conteúdo da RLR está licenciado sob Creative Commons Atribuição 4.0 Internacional

II [Lucien] lui demanda de lui raconter Le Petit Chaperon Rouge, et maman le prit sur ses genoux. Elle lui parla du loup et de la grand-mère du Chaperon Rouge, un doigt levé, souriante et grave. Lucien la regardait, il lui disait: «Et alors?», [...]; mais il ne l'écoutait pas, il se demandait si c'était bien sa vraie maman. Quand elle eut fini son histoire, il lui dit: «Maman, raconte-moi quand tu étais petite fille.»
Ele [Lucien] pediu a ela que the contasse Chapeuzinho Vermelho, e mamãe o colocou em seus joelhos. Ela lhe falou sobre o lobo e sobre a avó da Chapeuzinho Vermelho, com um dedo levantado, sorridente e grave. Lucien olhava ela e Ihe dizia: "E então?", [...]; mas ele não a escutava, perguntava-se se era mesmo a sua verdadeira mamãe. Quando ela acabou a história, ele the disse: "Mamãe, me conta de quando tu eras menininha."

De nouveau, quand nous avons relu nos premiers brouillons en portugais, quelque chose a sonné artificiel. Instruits par l'expérience, nous avons peu tardé à percevoir que notre malaise stylistique provenait de l'usage et de la répétition (quatre occurrences) de «/he», pronome pessoal oblíquo da terceira pessoa singular (pronom personnel complément d'objet indirect de la troisième personne du singulier). Dans le cinquième tableau, nous avons souligné les occurrences de "lui" dans le texte français, et celles de « Ihe » dans la version en portugais.

Tableau 5

\begin{tabular}{|c|c|}
\hline Texte source: deuxième extrait & Le premier jet de notre traduction \\
\hline $\begin{array}{l}\text { ॥l [Lucien] lui demanda de lui raconter Le Petit } \\
\text { Chaperon Rouge, et maman le prit sur ses genoux. } \\
\text { Elle lui parla du loup et de la grand-mère du } \\
\text { Chaperon Rouge, un doigt levé, souriante et } \\
\text { grave. Lucien la regardait, il lui disait: «Et } \\
\text { alors?», [...]; mais il ne l'écoutait pas, il se } \\
\text { demandait si c'était bien sa vraie maman. Quand } \\
\text { elle eut fini son histoire, il lui dit: «Maman, raconte- } \\
\text { moi quand tu étais petite fille.» }\end{array}$ & $\begin{array}{l}\text { Ele [Lucien] pediu a ela que } \underline{\text { hhe }} \text { contasse } \\
\text { Chapeuzinho Vermelho, e mamãe o colocou em } \\
\text { seus joelhos. Ela lhe falou sobre o lobo e sobre a } \\
\text { avó da Chapeuzinho Vermelho, com um dedo } \\
\text { levantado, sorridente e grave. Lucien olhava ela e } \\
\text { lhe dizia: "E então?", [...]; mas ele não a escutava, } \\
\text { perguntava-se se era mesmo a sua verdadeira } \\
\text { mamãe. Quando ela acabou a história, ele lhe } \\
\text { disse: "Mamãe, me conta de quando tu eras } \\
\text { menininha." }\end{array}$ \\
\hline
\end{tabular}

Pourquoi la répétition du pronom portugais « the » nous gênait-elle ? Pour deux raisons. D'une part, parce que le pronom portugais « Ihe » appartient au langage soutenu où il est, suivant le contexte, la marque du traitement de politesse, formel, respectueux, de convention ou solennel (HOUAISS, MICHAELIS). II détonne donc dans ce passage où le narrateur adopte le point de vue d'un enfant (qui, de par son âge, serait incapable de maîtriser l'usage de ce complément) et dont la tonalité est celle de l'intimité de ce petit enfant avec sa mère. D'autre part, l'utilisation de ce pronom en portugais étant rare à l'oral et limitée dans le langage soutenu, il est en 

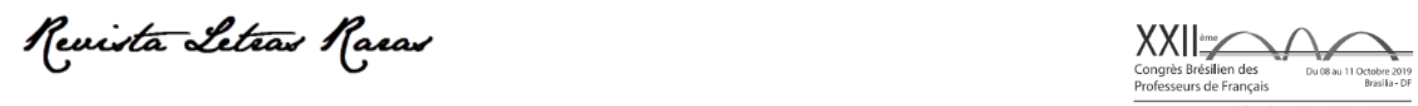

Actes du XXIlème Congrès brésilien des professeurs de français (dans Édition spéciale de la Revue Letras Raras 2020)

ISSN : 2317-2347 - v. 9, Dossier Spécial (2020)

Todo o conteúdo da RLR está licenciado sob Creative Commons Atribuição 4.0 Internacional

général supprimé par une ellipse ou remplacé par des substituts ou par la répétition de l'objet luimême.

Après ce constat, nous avons comparé les occurrences du pronom « lui » dans le texte français et celles du pronom correspondant dans notre brouillon en portugais. Nous avons remarqué que, dans celui-ci, nous avons systématiquement rendu par « Ihe » tous les « lui » du texte français (sauf un). D'où, de nouveau, une sensation d'inconfort, car l'ellipse de ce pronom étant courante en portugais, c'est son maintien qui sonne mal. Nous avons examiné une à une les mesures à prendre en tenant compte des deux observations formulées dans le paragraphe précédent.

Le résultat se trouve dans le tableau six. Dans la colonne de gauche, en français, nous avons souligné les occurrences de « lui », pronom personnel complément d'objet indirect de la troisième personne du singulier. Dans celle de droite, nous insérons le symbole du vide pour indiquer leur suppression.

Tableau 6

\begin{tabular}{|c|c|}
\hline Texte source: deuxième extrait & Nouvelle version de notre traduction \\
\hline $\begin{array}{l}\text { "I [Lucien] lui demanda de lui raconter Le Petit } \\
\text { Chaperon Rouge, et maman le prit sur ses genoux. } \\
\text { Elle lui parla du loup et de la grand-mère du } \\
\text { Chaperon Rouge, un doigt levé, souriante et grave. } \\
\text { Lucien la regardait, il lui disait: «Et alors ?», [...]; } \\
\text { mais il ne l'écoutait pas, il se demandait si c'était } \\
\text { bien sa vraie maman. Quand elle eut fini son } \\
\text { histoire, il lui dit: «Maman, raconte-moi quand tu } \\
\text { étais petite fille.» }\end{array}$ & $\begin{array}{l}\text { Ele [Lucien] pediu a ela que [Ø] } \\
\text { contasse Chapeuzinho Vermelho, e mamãe o } \\
\text { colocou em seus joelhos e [Ø] falou sobre o lobo e } \\
\text { sobre a avó da Chapeuzinho Vermelho, com um } \\
\text { dedo levantado, sorridente e grave. Lucien olhava } \\
\text { ela e [Ø] dizia: "E então?", [...]; mas ele } \\
\text { não a escutava, perguntava-se se era a sua } \\
\text { verdadeira mamãe. Quando ela acabou a história, } \\
\text { ele [Ø] disse: "Mamãe, me conta de quando tu eras } \\
\text { menininha." }\end{array}$ \\
\hline
\end{tabular}

Nous avons supprimé toutes les occurrences de "Ihe », au bénéfice de la fluidité de la version en portugais et de l'adéquation de la traduction à l'âge du protagoniste, au point de vue adopté par le narrateur et à la tonalité du passage.

Essayons maintenant de savoir ce qui s'est passé.

\section{Que s'est-il passé ?}



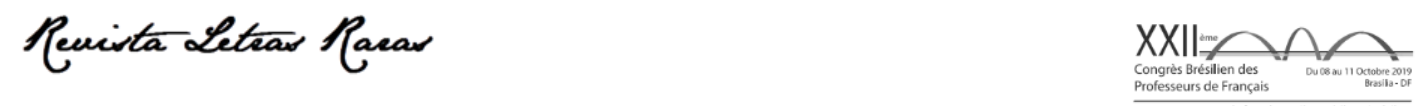

Actes du XXIlème Congrès brésilien des professeurs de français (dans Édition spéciale de la Revue Letras Raras 2020) ISSN : 2317-2347 - v. 9, Dossier Spécial (2020)

Todo o conteúdo da RLR está licenciado sob Creative Commons Atribuição 4.0 Internacional

Par quel cheminement, quels mécanismes avons-nous rendu tous (ou presque tous) les «il(s)/elle/lui » du français par « ele/ela/lhe »? Comment deux structures syntaxiques de la langue source (langue étrangère) ont-elles pu se manifester justement dans la langue cible du traducteur (sa langue forte, de référence)?

Pourquoi ? La langue étrangère a pénétré dans la langue maternelle du traducteur et l'a perturbée par un fait de langue naturel, connu, universel et toujours présent dans le bilinguisme : l'interférence. Phénomène « involontaire », il se produit « quand un sujet bilingue utilise dans une langue cible $A$ un trait phonétique, morphologique, lexical ou syntaxique caractéristique de la langue B» (DUBOIS, 2012, p. 252). En traduction, l'interférence est d'abord l'expression de l'énorme force d'attraction, du poids gigantesque du discours de la langue source.

La manifestation de l'interférence peut d'ailleurs avoir été facilitée parce que la vigilance du traducteur s'était assoupie, qu'il se trouvait en quelque sorte en pilotage automatique, qu'il se reposait sur un automatisme instinctif : coller au texte de départ. Cela arrive assez souvent, comme le signale Paulo Rónai: «[...] o erro nem sempre é fruto de ignorância. Basta um cochilo, um momento de cansaço, e o profissional de maior tarimba verterá remarquable por 'remarcável', e il cria por 'ele criou'» (RÓNAI, 1975, p. XIII). Moment de distraction, de baisse de l'attention d'autant plus compréhensible que le français et le portugais sont des langues sœurs dont les fortes ressemblances donnent fréquemment l'illusion qu'elles sont homologues, transparentes, alors qu'elles possèdent aussi de nombreuses différences, non des moindres. D'où l'occurrence d'interférences.

\section{Comment classifier ce type de difficulté ?}

L'interférence est la cause de certaines erreurs et bévues qui, en traduction, se manifestent dans le texte d'arrivée. De quel type de difficulté, de quel problème s'agit-il ? Dans la situation de traduction qui part du FLE pour arriver à une autre langue, elles sont souvent qualifiées de gallicismes (« incorrer em galicismos», HOUAISS). Le terme est utile et juste. Avec d'autres langues sources, on a des anglicismes, des hispanismes, etc. Y a-t-il un terme général, linguistique qui englobe cette famille d'-ismes?

Paulo Rónai, excellent traducteur et enseignant de la traduction, avait, dès 1975, annoncé, prévu les inadvertances analysées ci-dessus quand il mettait en garde contre «certas estruturas 

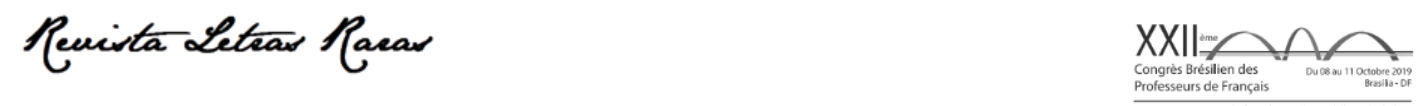

Actes du XXIlème Congrès brésilien des professeurs de français (dans Édition spéciale de la Revue Letras Raras 2020) ISSN : 2317-2347 - v. 9, Dossier Spécial (2020)

Todo o conteúdo da RLR está licenciado sob Creative Commons Atribuição 4.0 Internacional

sintáticas do francês, cujo simples decalque em português não só produz frases desajeitadas, mas muitas vezes chega a alterar-Ihes o sentido. Vejam-se os estragos que a tradução indistinta do pronome il pode produzir» (RÓNAI, 1975, p. XIII). On voit qu'il y propose un terme pour désigner ce type de problème : decalque (en français, « calque »).

Quelques années plus tard, Rónai retourne à ce sujet sans employer le terme «decalque». À la place, il utilise, comme synonymes, «tradução literal» («mesmo no campo técnico não existe tradução literal») et «tradução mecânica» (RÓNAI, 1984, p. 10-11). II y reprend la question de la traduction du pronom personnel «il» («a tradução mecânica de todos os 'il' pode dificultar a compreensão do enunciado») et cite quelques emplois verbaux impersonnels («il convient, il faut, il n'en est pas moins vrai» - tournures «sem analogia em português») pour faire comprendre «o absurdo contido na expressão 'tradução literal» (RÓNAI, in PORTINHO, 1984, p. 10-11).

Quel terme choisir pour désigner l'action de traduire en transportant mot à mot d'une langue à l'autre ? Considérant que la « traduction littérale » est généralement opposée à la « traduction libre » et que le contenu sémantique des deux syntagmes est variable, imprécis, polysémique, nous jugeons préférable d'éviter l'expression «traduction littérale». " Traduction mécanique » est une tentative séductrice, mais elle n'est pas d'usage courant, ne figure pas dans les dictionnaires et, surtout, le mot à mot n'est pas nécessairement du pilotage automatique, mécanique.

Nous avons alors fait des recherches sur le mot « decalque » et sur son équivalent français, "calque ». Elles ont été menées dans trois dictionnaires de la langue française (le Dictionnaire de l'Académie française - 9e édition, le Petit Robert et le Trésor de la langue française informatisé), dans quatre de la langue portugaise (AURÉLIO, AULETE, HOUAISS, MICHAELIS) et dans deux dictionnaires de linguistique (Martinet et Crystal). Dans ce travail, nous ne présentons que les résultats relatifs à la bibliographie en portugais.

Le mot « decalque » figure dans les quatre dictionnaires de portugais consultés, le mot portugais « calque »(dont la fréquence est rare) n'étant mentionné que dans le dictionnaire Aurélio (qui renvoie à «decalque»). Quant au mot « calco », c'est un terme propre au portugais du Portugal. En conséquence, nous employons seulement le mot « decalque » (en français, « calque »). 

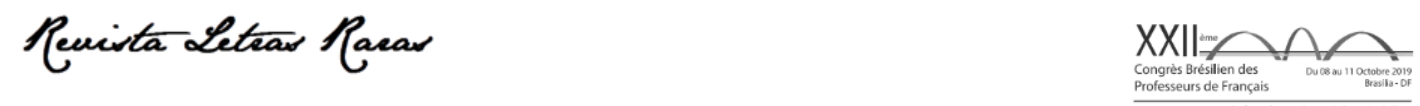

Actes du XXIlème Congrès brésilien des professeurs de français (dans Édition spéciale de la Revue Letras Raras 2020)

ISSN : 2317-2347 - v. 9, Dossier Spécial (2020)

Todo o conteúdo da RLR está licenciado sob Creative Commons Atribuição 4.0 Internacional

Dans nos recherches, nous avons constaté que le premier sens de « decalque »est toujours relatif à un procédé du dessin et des arts graphiques : "Transferência de uma imagem gráfica de uma superfície para outra, por compressão ou cópia» (AULETE, 2019).

Vient ensuite, par dérivation ou par extension, le sens de «cópia» (copie) ou «plágio» (plagiat) (AULETE, AURÉLIO, HOUAISS, MICHAELIS).

Finalement, toujours en dernière position, apparaît l'utilisation technique du mot comme terme de la linguistique. Aurélio (2014) et Houaiss (2011) précisent qu'il s'agit d'un type de «empréstimo» (emprunt, en français), c'est-à-dire l'«incorporação ao léxico de uma língua de um termo pertencente a outra língua». II y a plusieurs types d'emprunt et les divers types sont formés par «diferentes processos» (Houaiss, 2011). L'emprunt proprement dit (ou emprunt tout court) se produit tout simplement par la «reprodução do termo sem alteração de pronúncia e/ou grafia (know-how), ou com adaptação fonológica e ortográfica (garçom, futebol)» (Houaiss, 2011). Quant au «decalque» (calque), c'est un «empréstimo lexical em que se traduz para vocabulário nativo a formação estrangeira» (AURÉLIO, 2014). Le calque incorpore donc le mot étranger à la langue de réception par un processus d'assimilation, d'adaptation par traduction littérale faite absolument mot à mot, comme on le voit dans les exemples fournis par les dictionnaires consultés : trois mots composés, «cachorro-quente» (calque de l'anglais «hot-dog»), «arranha-céu» (calque de l'anglais «skyscraper»), «força-tarefa» (calque de l'anglais «task force»), et la locution «ter lugan» (calque du français «avoir lieu»).

La définition de David Crystal dans son dictionnaire de linguistique ne contredit en rien ce qui précède :

\footnotetext{
"Decalque: termo usado na linguística histórica e comparada para indicar um tipo de empréstimo em que os constituintes morfofonêmicos da palavra ou expressão emprestada são traduzidos item por item em morfemas equivalentes de outra língua. O exemplo 'Superhomem', do inglês Superman, ilustra esta 'tradução de empréstimo'." (2000, p. 74)
}

Arrivés à ce point, il faut signaler un apport important du dictionnaire Michaelis : des quatre dictionnaires en portugais consultés, il est le seul à mentionner que le mot « decalque » désigne aussi un «procedimento de tradução» (procédé de traduction) qui consiste à «traduzir literalmente sintagmas ou frases da língua original para a língua da tradução, como, por exemplo, task force = força-tarefa» (MICHAELIS, 2009). Autrement dit, le dictionnaire Michaelis informe que le terme "decalque" ne désigne pas seulement un type d'emprunt, mais aussi une technique de traduction. Le calque est en effet un procédé légitime de traduction. 

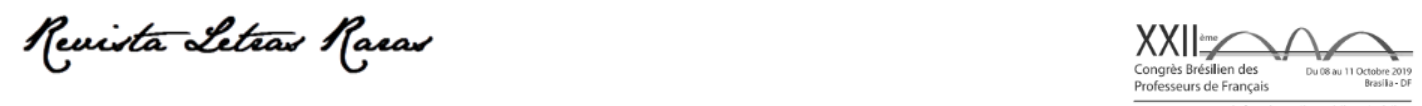

Actes du XXIlème Congrès brésilien des professeurs de français (dans Édition spéciale de la Revue Letras Raras 2020) ISSN : 2317-2347 - v. 9, Dossier Spécial (2020)

Todo o conteúdo da RLR está licenciado sob Creative Commons Atribuição 4.0 Internacional

Cependant, les mises en garde de Paulo Rónai sur les «estragos», les «frases desajeitadas» produits par le «simples decalque» (1975, p. XIII), son commentaire sur «0 absurdo contido na expressão 'tradução literal'» $(1984$, p. 11) avertissent que le calque ne doit pas être vu comme une recette magique, infaillible et toujours pertinente.

De tout ce qui précède, nous concluons que, comme tous les autres procédés de traduction, le calque peut, suivant les cas, les contextes, être approprié, utile ou non. II ne doit donc pas être utilisé indistinctement, sans critères. De sorte que, pour plus de clarté, nous suggérons l'usage du mot « decalque » (en français, calque) pour désigner le procédé de traduction (ainsi que le type d'emprunt respectif, l'emprunt par calque) et l'expression «calque fautif» ou «calque erroné» pour référer aux emplois inadéquats, inappropriés du procédé de traduction.

\section{En guise de conclusion}

Dans cet article, nous nous sommes penchés sur les occurrences de deux difficultés de nature lexico-syntaxique qui se sont manifestées pendant notre traduction, du français en portugais, de morceaux de «L'Enfance d'un chef », de Sartre. Après la présentation du projet au sein duquel nous avons développé cette réflexion, notre travail a parcouru quatre parties ou étapes principales.

Deux étapes ont été consacrées à l'étude des deux difficultés lexico-syntaxiques : d'une part, la traduction des pronoms personnels sujets de la troisième personne du singulier ; d'autre part, la traduction des pronoms personnels compléments d'objet indirect. Chacun des deux extraits choisis de la nouvelle de Sartre était un bon échantillon, car il contenait une quantité d'occurrences des deux difficultés citées. Nous avons expliqué, pas à pas, en illustrant chaque cas avec trois tableaux, comment, à partir du premier jet, nous avons décelé qu'il y avait des problèmes, des inadéquations dans les brouillons de nos versions en portugais et comment nous sommes arrivés à des solutions satisfaisantes pour chaque groupe de difficulté.

Dans la troisième étape, nous avons mené une réflexion sur les causes qui permettent ou provoquent la manifestation de ces difficultés, en particulier sur les interférences qui sont inhérentes à toute situation de bilinguisme et menacent constamment, mais nous avons aussi 

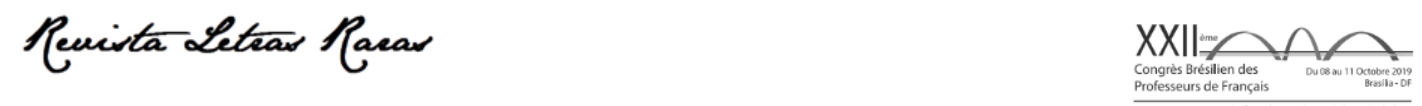

Actes du XXIlème Congrès brésilien des professeurs de français (dans Édition spéciale de la Revue Letras Raras 2020) ISSN : 2317-2347 - v. 9, Dossier Spécial (2020)

Todo o conteúdo da RLR está licenciado sob Creative Commons Atribuição 4.0 Internacional

insisté sur la vigilance qui doit nécessairement accompagner le traducteur à tout moment pour justement contrecarrer le péril des interférences.

Finalement, dans la quatrième et dernière étape de ce travail, nous nous sommes consacrés à une analyse théorique visant à permettre d'obtenir une définition (ou dénomination) du type (ou classe) de problème auquel appartiennent les deux difficultés concrètes étudiées. Nous avons consulté plusieurs ouvrages en nous efforçant de comparer l'utilité de termes comme traduction littérale, traduction machinale et calque. Au terme de ces recherches, nous avons conclu que le mot calque (en portugais, « decalque ») était le plus adéquat, mais que le procédé (ou technique) du calque doit être manié avec précautions: ce n'est pas une recette infaillible. Suivant le contexte, le cas concret, il peut être employé ou ne doit pas l'être. Et s'il est mal employé, le résultat sera un calque fautif (erroné).

\section{Références}

AULETE, F.; VALENTE, A. Dicionário online Caldas Aulete. São Paulo: Lexikon Editora Digital, 2019. Disponível em: http://www.aulete.com.br/ Acesso em: 20 outubro de 2020.

BATH, S.; BIATO, O.. Les faux amis e outras peculiaridades da língua francesa para uso dos brasileiros. Brasília: Editora da UnB, col. "Humanidades", 1998.

BUARQUE DE HOLANDA, A. Minidicionário Aurélio versão eletrônica. Curitiba: Positivo, 2014.

CRYSTAL, D. Dicionário de linguística e de fonética. Tradução e adaptação de Maria Carmelita de Pádua Dias. São Paulo: Cultrix, 2000.

Dicionário online Michaelis. São Paulo: Melhoramentos, 2009. Disponível em: https://michaelis.uol.com.br/ Acesso em: 20 outubro de 2020

DUBOIS, J. et alii. Dictionnaire de linguistique et des sciences du langage. Paris: Larousse, 2002.

DURIEUX, C. «La difficulté en traduction». Revue des lettres et de traduction, 1999, n5. Université du Saint-Esprit de Kaslik (Liban). P. 31-34. Disponível em: http://documents.irevues.inist.fr/bitstream/handle/2042/41863/1999_5_31\%2034.pdf?sequence=3 \&isAllowed=y Acesso em: 20 de outubro de 2020.

HOUAISS, A. Dicionário Houaiss do português. CD-ROM. Rio de Janeiro: Objetiva, 2011.

MOUNIN, G. Problèmes théoriques de la traduction. Paris : Gallimard, coll. "Bibliothèque des idées", 1971.

PORTINHO, W. M. (Org.). A tradução técnica e seus problemas. São Paulo: Álamo, 1984.

RÓNAI, P. Guia prático da tradução francesa. $2^{a}$ ed., rev. e ampliada. Rio de Janeiro: Educom, 1975. 

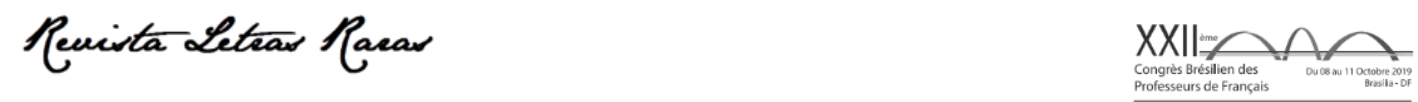

Actes du XXIlème Congrès brésilien des professeurs de français (dans Édition spéciale de la Revue Letras Raras 2020)

ISSN : 2317-2347 - v. 9, Dossier Spécial (2020)

Todo o conteúdo da RLR está licenciado sob Creative Commons Atribuição 4.0 Internacional

RÓNAI, P. "Problemas gerais da tradução". In: PORTINHO, Waldivia Marchiori (org.). A tradução técnica e seus problemas. São Paulo: Álamo, 1984, pp. 1-15.

RÓNAI, P. A tradução vivida. Rio de Janeiro: Educom, 1976.

RÓNAI, P. Escola de Tradutores. 6ª ed. Rio de Janeiro: Nova Fronteira, 1977.

SARTRE, J-P. (1939) Le Mur. Paris : Gallimard, coll. Folio, 2013.

SILVA, G. J. ; PONGE, R. « Les expressions idiomatiques et les difficultés de compréhension et de traduction du FLE». In: Synergies Brésil n 10 - 2012, pp. 113-122. Disponível em: https://gerflint.fr/Base/Bresil10/da_silva.pdf. Acesso em: 31 de outubro de 2020.

VINAY J.-P. e DARBELNET J. Stylistique comparée du français et de l'anglais : méthode de traduction. Coll. « Bibliothèque de stylistique comparée ». Nouvelle édition revue et corrigée. Paris: Didier, 1972.

XATARA, C.; OLIVEIRA, W. L. de. Dicionário de falsos cognatos francês português/portuguêsfrancês. $2^{\mathrm{a}}$ ed., revista e ampliada. São Paulo: Editora de Cultura, 2008. 